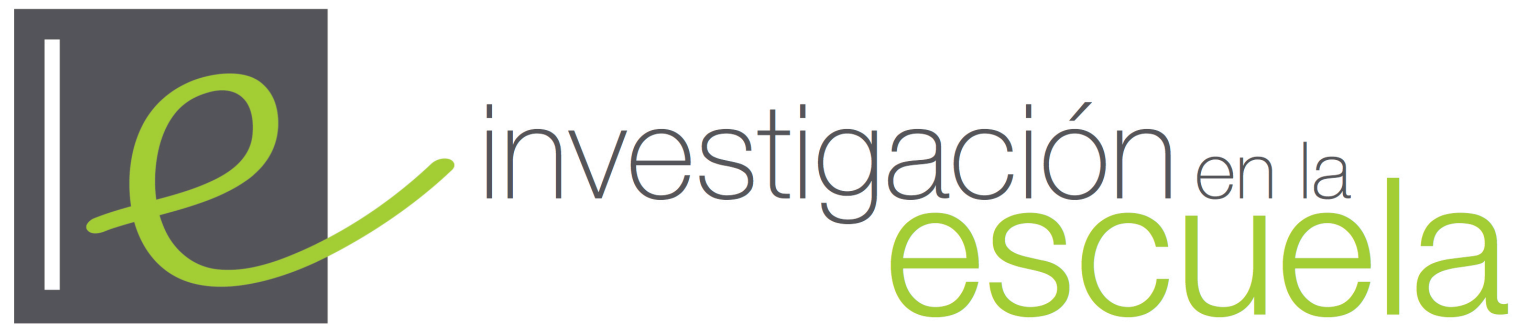

Revista internacional de investigación e innovación educativa

\title{
Indagando en el aula de ciencias: cómo progresan los métodos empleados por los docentes. Un estudio de caso
}

\author{
Julià Hinojosa Lobato y Neus Sanmartí i Puig \\ Universidad Autónoma de Barcelona \\ España
}

Citación: Hinojosa Lobato, J. y Sanmartí i Puig, N. (2019). Indagando en el aula de ciencias: cómo progresan los métodos empleados por los docentes. Un estudio de caso. Investigación en la Escuela, 99, 15-31. doi: http://dx.doi.org/10.12795/IE.2019.i99.02

Resumen: En este artículo se presenta una propuesta de los estadios posibles en un proceso de cambio docente, obtenidos de forma empírica, en relación a la aplicación de procesos de indagación en el aula, a partir de un programa de formación permanente implementado en una escuela de Barcelona. Se pretende que los docentes promuevan una actividad científica indagatoria que dé importancia a la argumentación y la modelización aplicando procesos de indagación. Aunque el proyecto abarca todo el profesorado, de los tres a los 18 años en una escuela de cuatro líneas, en este escrito nos centramos en el de $5^{\circ}$ curso de primaria. Para el estudio de su evolución se ha triangulado información a partir del diseño de las actividades realizadas por los maestros, las producciones elaboradas por los alumnos, y los datos recogidos en el cuaderno de campo donde se anotan preguntas, respuestas y reflexiones de los maestros. Se han identificado diferentes subprocesos asociados, cómo se va progresando en cada uno de ellos y las dificultades de consolidación de esta metodología.

Palabras clave: "Formación permanente"; "modelización”; “argumentación”; "indagación”. 
Investigating in the science classroom: how the methods used by teachers are progressing. A case study

Abstract: This article presents a proposal of the possible stages in a process of teacher change, obtained empirically, in relation to the application of inquiry processes in the classroom, based on a continuous teacher professional development program implemented in a school in Barcelona. It is intended that teachers promote an inquiry scientific activity that gives importance to argumentation and modelling by applying inquiry processes. Although the project covers all teachers, from early years up to high school in a school of four lines, in this paper we focus on the fifth year of primary school. For the study of its evolution, information has been triangulated based on the design of the activities carried out by the teachers, the productions made by the students, and the data collected in the field notebook where questions, answers and reflections of the teachers are recorded. Different associated subprocesses have been identified, how progress is being made in each of them and the difficulties of consolidating this methodology.

Key words: "Teacher training"; "inquiri"; "modelling"; "argumentation".

\section{Enquêter dans la classe de sciences : comment les méthodes utilisées par les enseignants progressent Une étude de cas}

Resumè: Cet article présente une proposition des étapes possibles d'un processus de changement d'enseignant, obtenu de manière empirique, en relation avec l'application de processus d'investigation en classe, sur la base d'un programme de «formation permanente » mis en œuvre dans une école de Barcelona. Il est prévu que les enseignants promeuvent une activité scientifique d'investigation qui donne une importance à l'argumentation et à la modélisation en appliquant des processus d'investigation. Bien que le projet couvre tous les enseignants, de trois à dix-huit ans dans une école de quatre lignes, nous nous concentrons dans cet article sur la cinquième année d'école primaire. Pour l'étude de son évolution, les informations ont été triangulées sur la base de la conception des activités réalisées par les enseignants, des productions élaborées par les étudiants et des données collectées dans le cahier de terrain où sont consignées les questions, réponses et réflexions des enseignants. Différents sous-processus associés ont été identifiés, les progrès réalisés dans chacun d'entre eux et les difficultés de consolidation de cette méthodologie.

Mots-clés: "Formation permanente"; "modélisation"; "argumentation"; "enquête".

\section{Introducción y objetivos}

El paso de un aula con propuestas didácticas de tipo transmisivo a otra en la que se promueve que los alumnos se planteen preguntas e indaguen sobre cómo darles respuesta, no es fácil ni rápido. Requiere cambios: a) conceptuales -qué se entiende por 'investigar', por una pregunta investigable, por prueba, por argumento... y, en general, por ciencia-; b) metodológicos -cómo gestionar el grupo-clase y el tiempo, cómo ayudar a establecer relaciones entre los trabajos prácticos y las ideas teóricas, cómo evaluar, etc.- y c) actitudinales, entre otros los relacionados con la inseguridad que genera cualquier cambio. Todo ello, comporta cambiar rutinas que están muy establecidas, ya que los docentes las vivieron en toda su etapa de aprendices y son las que observan en sus compañeros.

Para promover su evolución no son útiles los tradicionales cursos de formación ya que se han de generar procesos de aprendizaje compartido, largos en el tiempo, que promuevan una reflexión colectiva sobre la práctica y posibiliten profundizar en los marcos teóricos que la sustentan (Capps, Crawford y Constas, 2012; Coenders y Terlouw, 2015). 
Este artículo recoge parte de un estudio realizado en el marco de un proyecto que tiene como finalidad promover entre los profesores de una escuela de Barcelona una estrategia didáctica en la que se aprenda ciencia y sobre ciencia, es decir, se construyan conocimientos significativos de ciencia y de los procesos que utiliza la ciencia para generarlos. Para ello se ha asesorado al equipo de profesores a través de un espacio de formación e innovación fundamentado en la reflexión conjunta y permanente (Copello y Sanmartí, 2001; Esteve, 2013; Vázquez et al., 2013).

El objetivo específico de este trabajo es presentar una propuesta de etapas o estadios en la progresión de la aplicación de una metodología para el aprendizaje de las ciencias basada en la indagación y modelización. Esta propuesta se plantea a partir de analizar empíricamente los cambios en la práctica de tres maestras y un maestro de $5^{\circ}$ de primaria, a partir de un proceso de asesoría desarrollado en equipo a lo largo de dos cursos. Se han caracterizado cambios en base a diferentes subprocesos que pueden caracterizar dicha metodología, y se han identificado algunas de las dificultades a afrontar para promover el cambio en las ideas y en las prácticas de los docentes.

\section{Marco teórico}

Toda propuesta docente se inscribe en un marco teórico de forma explícita o implícita, que tiene fundamentos epistemológicos (qué ciencia aprender), psicológicos (cómo se cree que se aprende), pedagógicos (cómo promover su aprendizaje) y sociológicos (para qué aprenderla) (Coll, 1994). Para alcanzar los objetivos de la enseñanza de las ciencias que cada docente considera relevantes, se seleccionan determinados contenidos, se programan actividades, se preparan materiales y recursos, se organiza el trabajo en el aula..., es decir, se toman una serie de decisiones sobre qué enseñar y cómo hacerlo. Estas decisiones y estrategias responden a un modelo didáctico o a una mezcla de distintos modelos.

Como señalan Porlán et al., (2010), el cambio en las concepciones y prácticas del profesorado no es fácil, pero es posible. Requiere tener en cuenta cómo se va progresando en la superación de los obstáculos y las posibles transiciones, sabiendo que una de las dificultades más importantes a superar es que dichas prácticas responden a una visión epistemológica de la ciencia, pero buena parte de los docentes no son conscientes de ella y es difícil que puedan analizarla.

En esta línea, Vázquez et al. (2013), postulan la Hipótesis de la Complejidad para argumentar que, en la evolución de las ideas y prácticas del profesorado, interaccionan aspectos ideológicos, formativos, contextuales, epistemológicos, curriculares y emocionales, y que el cambio proviene de tenerlas todas en cuenta. En su estudio comprobaron que los obstáculos se pueden superar cuando, en el marco de un grupo de trabajo, se da un soporte profesional, social y emocional, orientado a promover la revisión de los propios pensamientos y actuaciones. A partir de reflexiones compartidas o en la soledad de la introspección, afloran las concepciones que sustentan las prácticas de aula, se movilizan e incluso se remueven. De aquí la importancia de los grupos de innovación para el desarrollo profesional del profesorado (Mellado et al., 2006), ya que posibilitan construir conocimientos relacionados con los problemas que más afectan a sus componentes gracias a la reflexión compartida y a la discusión crítica de prácticas reales. Esta colaboración permite, además, encauzar las emociones negativas que se puedan generar, a través del soporte afectivo del grupo.

Para promover el aprendizaje científico del alumnado se generan actividades diversas, pero muchas de las tareas de investigación que se promueven en los estudiantes no reflejan los atributos centrales de un razonamiento científico. Los procesos cognitivos necesarios para tener éxito en muchas tareas escolares a menudo son cualitativamente diferentes de los procesos cognitivos necesarios para participar en una investigación científica real. Por ejemplo, en muchas de las prácticas de Aprendizaje Basado en Proyectos se pide a los estudiantes que "investiguen” y de hecho 
sólo se promueve que busquen informaciones y las copien. Por ello, es necesario revisar las prácticas de instrucción habituales y desarrollar una variedad de nuevas tareas que incorporen aspectos propios de un razonamiento auténtico (Chinn y Malhotra, 2002).

Actualmente, dentro del abanico de metodologías por indagación, diferentes enfoques hacen hincapié en la indagación basada en la modelización de fenómenos (IBM) (Windschitl, Thompson y Braaten, 2008). Desde este punto de vista, se considera la enseñanza como el conjunto de acciones que promueve el profesorado para favorecer el proceso de indagación-modelización que realizan los estudiantes con la finalidad de "dar sentido" a los hechos del mundo (Sanmartí, 2002). La finalidad es la construcción de representaciones de la realidad (modelos) con el objetivo de explicarla y de hacer predicciones, que se evalúan y reajustan cuando se ponen en práctica (Schwarz et al., 2009).

Hacer ciencia es, por tanto, llevar a cabo una actividad en la cual la indagación, la modelización y la argumentación se entrelazan para una reconstrucción de los fenómenos y enseñar ciencias. Como indica Jiménez-Aleixandre (2000), conlleva mediar en el proceso de aprendizaje, a partir tanto de la planificación y organización de actividades relevantes, como de la gestión del trabajo individual y en equipo, y de la intervención en determinadas etapas de dicho proceso. Dicha intervención promueve la explicitación de ideas o modelo mental, búsqueda de pruebas y construcción de nuevas ideas científicas, reestructuración (evaluación-regulación) de conocimientos en función de las pruebas y de la perspectiva científica, y aplicación de las ideas a nuevos contextos o tareas (Couso, 2014).

Como indica Osborne (2014), el objetivo principal de una práctica científica es desarrollar el conocimiento y la comprensión de los estudiantes, y cómo dicha práctica contribuye a saber qué sabemos y a construir conocimientos fiables. Ello implica situar las habilidades de crítica y evaluación en el centro del aprendizaje de la ciencia.

\section{Dinámica del asesoramiento}

Basándonos en estos enfoques, se ha aplicado una metodología de formación docente compartida o, mejor dicho, de asesoramiento para una reflexión conjunta entre el profesorado. Con ello se persiguen cuatro grandes metas (Valcárcel y Sánchez, 2000):

- Mejorar el conocimiento de los profesores en relación con la materia que enseñan, tanto del conocimiento del contenido como del conocimiento didáctico del contenido.

- Cambiar las concepciones y prácticas docentes hacia enfoques constructivistas que tengan en cuenta la indagación basada en la modelización.

- Capacitar a los profesores como diseñadores de proyectos e investigadores de su propia actuación en el aula.

- Desarrollar actitudes y prácticas docentes más colaborativas, críticas y autónomas.

Para avanzar en esta línea se ha abandonado el papel de formadores expertos e infalibles, que explican soluciones magistrales de otros para que los maestros las apliquen en el aula. En estas prácticas de formación, aunque se fundamenten teóricamente, las nuevas propuestas quedan reducidas al plano del discurso y no acostumbran a incorporarse a la práctica cotidiana de las clases o sólo se integran las técnicas o recursos concretos, sin cambiar las concepciones (Copello y Sanmartí, 2001). En cambio, se ha adoptado la función mediadora, y el formador asumió un papel de estímulo y colaboración en los procesos de discusión y argumentación, ayudando a crear espacios de formación, innovación y reflexión. Estos espacios se generaron a partir de desafiar a los profesores para que examinen sus conocimientos y creencias, y reflexionen sobre su práctica docente, todo con la finalidad de superar los obstáculos y encontrar soluciones a los problemas detectados (Capps, Crawford y Constas, 2012; Imbernón, 2007; Porlán, 2016).

Para ello se ha promovido que cada docente, trabajando en el espacio de sus concepciones y 
de sus prácticas, tome conciencia de ellas y adopte decisiones que, a su vez, generen mejoras en el aprendizaje de sus alumnos. Se ha trabajado en la línea de propuestas similares, como son las de Formación de Profesores para Investigar la Práctica (FOPIP) (Porlán, Rivero y Solís, 2011), o las de Práctica Reflexiva (Esteve, 2013), con la finalidad de ayudar a los profesores a elaborar modelos metodológicos conscientes y de cierta complejidad, con capacidad para orientar una práctica innovadora y profesionalizada.

El primer paso fue realizar una actividad común para explorar la situación inicial, es decir, para saber cómo se aplicaban procesos para la enseñanza de las ciencias en distintos niveles educativos. El reto que se propuso fue indagar sobre la flotación de los cuerpos, actividad que posibilitaba realizarla a diferentes niveles y generar modelos explicativos progresivamente más complejos (Garrido y Couso, 2017). Realizamos un estudio cualitativo de los resultados de esta exploración a partir de los informes realizados por los alumnos y de las reflexiones expresadas por los maestros y profesores de ciencias recogidas a partir de un guion que les pedía que explicaran cómo prepararon la actividad y cómo la aplicaron, y una reflexión después de realizarla. Se constató que, en algunos casos, no se comparte con el alumnado los objetivos de la actividad, ni se dedica tiempo a explicarlos y a justificarlos; no se pide que se argumenten las respuestas, ni que se debatan (interviene sólo el primer estudiante que dice que lo sabe); no se deja que los aprendices intervengan en el diseño experimental, ni que se planteen preguntas, y tampoco se controlan las variables.

El segundo paso consistió en realizar encuentros con los diferentes equipos de profesores (tres, en función de las etapas educativas), en los que se revisó conjuntamente la actividad realizada para reforzar aquello que fuera válido y, al mismo tiempo, reflexionar sobre lo que convendría replantear. A partir de este proceso, se discutió cómo se podría reorientar el proceso aplicado y también la estructura general de una actividad indagativa (Hinojosa y Sanmartí, 2016).

Posteriormente, en función de preguntas que habían surgido en las clases y las necesidades manifestadas por los docentes, se fueron abordando otras temáticas. Primero, sólo planteando alguna actividad puntual de indagación alrededor de alguna de las preguntas, que conllevó la realización de tres reuniones con cada equipo (antes, durante y después de aplicarla). Ejemplos de preguntas fueron: ¿Cómo encontramos el agua en la Naturaleza?, que posibilitó hablar de la modelización de los cambios en la materia, o ¿Cómo medir el tiempo sin un reloj?, que conllevó hablar de instrumentos para la medición de magnitudes. Poco a poco, a medida que el equipo ganaba confianza, se planificaron unidades didácticas más complejas e incluso proyectos: ¿Por qué se ha muerto la planta? (modelizar la concepción de ser vivo); ¿Por qué la mano necesita el corazón? (modelizar una visión sistémica del cuerpo humano); ¿Por qué cuando enciendo una linterna en una habitación oscura veo las cosas? (modelo de luz).

Paralelamente también se incidió en el análisis de las preguntas orientadas a promover en el alumnado la reflexión sobre los procesos experimentales que aplicaban. Se consensuó con los profesores una "base de orientación" con esta finalidad a partir de ayudar a pensar en: ¿qué tenemos? ¿qué hacemos? ¿qué crees que pasará? ¿qué ha pasado? y ¿por qué ha pasado?

De las diferentes propuestas, cada equipo analizaba la programación planteada por los maestros y los productos realizados por los alumnos. La intención de esta reflexión crítica era que los mismos profesores colaboraran en el diagnóstico y encontraran soluciones a los problemas detectados, proponiendo mejoras en los procesos de enseñanza-aprendizaje aplicados.

\section{Metodología de la investigación}

Este estudio de caso busca identificar cómo evolucionan las metodologías aplicadas por un equipo de cuatro maestros (tres maestras y un maestro, todos con más de 20 años de experiencia 
excepto una de las maestras que tiene sólo algo más de cinco años) de $5^{\circ}$ de primaria a lo largo de los dos primeros cursos de la formación. Se ha escogido este grupo porque ya desde el inicio manifestaron un interés por mejorar sus clases en la línea de trabajo que se les proponía. Como remarca Hargreaves (1999), la buena enseñanza no sólo radica en el conocimiento de habilidades y competencias, también se alimenta con la pasión, el desafío, la creatividad y la alegría.

Para el estudio de su evolución se partió de tres tipos de datos con la finalidad de triangular fuentes de información: el diseño de las actividades realizadas por los maestros, las producciones elaboradas por los alumnos, y los datos recogidos en el cuaderno de campo donde se anotaban preguntas, respuestas y reflexiones del equipo de maestros. Se asesoró aproximadamente una experiencia por trimestre y equipo.

Para el análisis de estos datos se han diseñado un conjunto de indicadores (ver tabla 1), en función de la propuesta de Simarro, Couso y Pintó (2013) sobre los aspectos a tener en cuenta en la modelización, propuesta que incide especialmente en las relaciones entre el "mundo de lo observable" y el "mundo de las ideas" (ver figura 1). Además, se han incluido dos nuevos ítems que se relacionan con la motivación del alumnado y con la autoevaluación-metacognición, dada la importancia evidenciada en distintos estudios de utilizar la evaluación como herramienta de aprendizaje (Romero-Ariza, 2017).

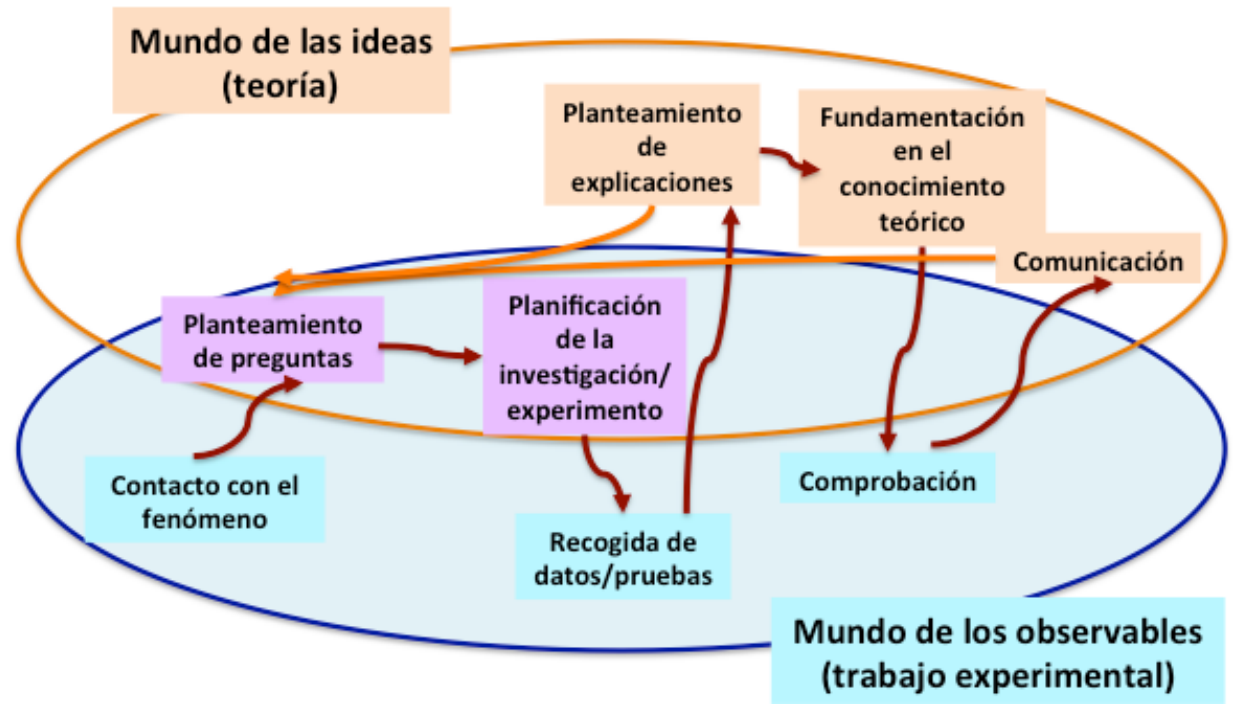

Figura 1. Propuesta de referente para el análisis de datos de la IBM. Adaptado de: Simarro, Couso y Pintó (2013)

Estos indicadores se han aplicado al análisis de los datos recogidos. Los dos autores del artículo han contrastado sus valoraciones y en el caso de discrepancias las han discutido hasta llegar al consenso. Este fue el caso, por ejemplo, del análisis del contexto de la indagación. Fue determinante acordar que el criterio para valorar la progresión no sólo se redujera a considerar si el escogido era relevante y significativo, sino también si daba sentido a todo el proceso de indagación. Por tanto, se analizó la planificación de los maestros, se contrastó con las producciones de los alumnos, y fueron muy clarificadoras las notas de campo que posibilitaban comparar alguna expresión inicial del tipo "¿Con diez minutos es suficiente, ¿no?”, con otras finales: “Asípues, hemos de pensar una situación cercana (local) y que sea valiosa para los alumnos y para lo que queremos enseñar... ino va a ser fácil!’”. 


\section{Resultados y reflexiones}

Se ha identificado, de forma inductiva, una serie de indicadores (véase tabla 1) que buscan recoger diferentes etapas de progreso de los docentes. Se ha caracterizado el punto de partida y dos estadios evolutivos que recogen los avances observados. También se indica un cuarto estadio con indicadores esperados en una etapa avanzada del proceso de formación.

Tabla 1

Progresión en distintos subprocesos aplicados por los maestros

\begin{tabular}{|c|c|c|c|c|}
\hline & $\begin{array}{l}\text { ESTADIO } \\
\text { INICIAL }\end{array}$ & ESTADIO 1 & ESTADIO 2 & $\begin{array}{l}\text { ESTADIO DE } \\
\text { REFERENCIA }\end{array}$ \\
\hline $\begin{array}{l}\text { Contexto de la } \\
\text { indagación / } \\
\text { contacto con el } \\
\text { fenómeno }\end{array}$ & Inexistente. & $\begin{array}{l}\text { Anécdota } \\
\text { inicial. }\end{array}$ & $\begin{array}{l}\text { Se plantea, pero } \\
\text { se acaba } \\
\text { abandonando. }\end{array}$ & $\begin{array}{l}\text { Actividad inicial que } \\
\text { dé sentido a todo el } \\
\text { proceso de indagación. }\end{array}$ \\
\hline $\begin{array}{l}\text { Planteamiento y } \\
\text { gestión de las } \\
\text { preguntas }\end{array}$ & $\begin{array}{l}\text { Las plantea el } \\
\text { docente y } \\
\text { responde el } \\
\text { primer alumno } \\
\text { que tiene una } \\
\text { propuesta de } \\
\text { predicción de } \\
\text { lo que puede } \\
\text { pasar sin dar } \\
\text { tiempo a que } \\
\text { los demás } \\
\text { activen sus } \\
\text { conocimientos } \\
\text { iniciales. }\end{array}$ & $\begin{array}{l}\text { Las plantea el } \\
\text { docente y se } \\
\text { da tiempo a } \\
\text { todos los } \\
\text { alumnos para } \\
\text { pensar en } \\
\text { posibles } \\
\text { respuestas, } \\
\text { predicciones } \\
\text { o hipótesis. } \\
\text { Pero no se } \\
\text { pide que las } \\
\text { argumenten ni } \\
\text { se debaten. }\end{array}$ & $\begin{array}{l}\text { Se pide que los } \\
\text { alumnos } \\
\text { planteen } \\
\text { preguntas. Se } \\
\text { anima a que } \\
\text { piensen en } \\
\text { posibles } \\
\text { predicciones o } \\
\text { hipótesis } \\
\text { individualmente } \\
\text {, para luego } \\
\text { contrastarlas en } \\
\text { pequeño grupo. } \\
\text { Finalmente se } \\
\text { comparten en } \\
\text { gran grupo, } \\
\text { pero no se } \\
\text { debate sobre su } \\
\text { posible } \\
\text { idoneidad para } \\
\text { indagar. }\end{array}$ & $\begin{array}{l}\text { Se analizan las } \\
\text { preguntas de los } \\
\text { alumnos y las del } \\
\text { docente y las } \\
\text { predicciones o } \\
\text { hipótesis. Se debate su } \\
\text { posible idoneidad para } \\
\text { indagar en base a los } \\
\text { conocimientos que se } \\
\text { tienen y a experiencias } \\
\text { anteriores. }\end{array}$ \\
\hline
\end{tabular}




\begin{tabular}{|c|c|c|c|c|}
\hline $\begin{array}{l}\text { Diseño de la } \\
\text { investigación/ } \\
\text { experimento para } \\
\text { recoger pruebas }\end{array}$ & $\begin{array}{l}\text { Seleccionado } \\
\text { por el docente } \\
\text { con la finalidad } \\
\text { de observar el } \\
\text { fenómeno. No } \\
\text { hay control } \\
\text { estricto de } \\
\text { variables. Son } \\
\text { de ensayo- } \\
\text { error. }\end{array}$ & $\begin{array}{l}\text { Seleccionado } \\
\text { por el docente } \\
\text { (a veces, por } \\
\text { los propios } \\
\text { alumnos) } \\
\text { buscando la } \\
\text { espectacularid } \\
\text { ad y la } \\
\text { 'motivación'. } \\
\text { (“iParece magia! } \\
\text { y se lo pasan } \\
\text { muy bien”). } \\
\text { No hay } \\
\text { control } \\
\text { estricto de } \\
\text { variables. }\end{array}$ & $\begin{array}{l}\text { Diseñado por el } \\
\text { docente con la } \\
\text { finalidad de } \\
\text { comprobar } \\
\text { predicciones } \\
\text { bastante } \\
\text { superficiales. } \\
\text { No se explicitan } \\
\text { los procesos } \\
\text { (¿qué está } \\
\text { pasando?), ni su } \\
\text { relación con la } \\
\text { búsqueda de } \\
\text { pruebas en } \\
\text { relación al } \\
\text { modelo inicial. }\end{array}$ & $\begin{array}{l}\text { Concebidos y } \\
\text { razonados por los } \\
\text { alumnos, y orientados } \\
\text { a buscar pruebas no } \\
\text { sólo de la predicción o } \\
\text { hipótesis planteada } \\
\text { sino también del } \\
\text { modelo teórico o ideas } \\
\text { que la sustentan. }\end{array}$ \\
\hline $\begin{array}{l}\text { Recogida y } \\
\text { explicitación de } \\
\text { las pruebas } \\
\text { obtenidas }\end{array}$ & $\begin{array}{l}\text { Datos } \\
\text { cualitativos, } \\
\text { orales y poco } \\
\text { específicos. La } \\
\text { recogida es } \\
\text { poco } \\
\text { sistemática. }\end{array}$ & $\begin{array}{l}\text { Datos } \\
\text { cualitativos } \\
\text { dibujados. La } \\
\text { recogida es } \\
\text { algo } \\
\text { sistemática. }\end{array}$ & $\begin{array}{l}\text { Datos } \\
\text { cualitativos y } \\
\text { alguno } \\
\text { cuantitativo. Se } \\
\text { organizan en } \\
\text { alguna tabla o } \\
\text { cuadro. }\end{array}$ & $\begin{array}{l}\text { Se cuantifica cuando } \\
\text { es necesario. Se } \\
\text { organizan en tablas y } \\
\text { gráficos de forma que } \\
\text { favorece la } \\
\text { comparación y el } \\
\text { análisis. }\end{array}$ \\
\hline $\begin{array}{l}\text { Planteamiento de } \\
\text { explicaciones y } \\
\text { argumentos en } \\
\text { base a modelos } \\
\text { teóricos }\end{array}$ & $\begin{array}{l}\text { Inexistente, no } \\
\text { se tiene en } \\
\text { cuenta. }\end{array}$ & $\begin{array}{l}\text { El docente da } \\
\text { la explicación } \\
\text { en base a } \\
\text { alguna idea- } \\
\text { definición. } \\
\text { No se tienen } \\
\text { en cuenta las } \\
\text { ideas previas. }\end{array}$ & $\begin{array}{l}\text { Se recuerdan las } \\
\text { ideas ya } \\
\text { construidas } \\
\text { pero de forma } \\
\text { aislada sin } \\
\text { vincularlas a } \\
\text { algún modelo } \\
\text { teórico ni } \\
\text { tampoco se } \\
\text { relacionan con } \\
\text { las pruebas } \\
\text { recogidas para } \\
\text { interpretarlas. }\end{array}$ & $\begin{array}{l}\text { Movilizar el modelo } \\
\text { teórico de referencia, } \\
\text { conectándolo con las } \\
\text { ideas previas- } \\
\text { predicciones y } \\
\text { relacionándolo con las } \\
\text { pruebas obtenidas. }\end{array}$ \\
\hline
\end{tabular}




\begin{tabular}{|c|c|c|c|c|}
\hline $\begin{array}{l}\text { Revisión de } \\
\text { referencias } \\
\text { bibliográficas }\end{array}$ & Anárquica. & $\begin{array}{l}\text { No responde } \\
\text { a preguntas } \\
\text { significativas } \\
\text { en relación } \\
\text { con el modelo } \\
\text { teórico. Se } \\
\text { utiliza para } \\
\text { copiar } \\
\text { informaciones }\end{array}$ & $\begin{array}{l}\text { Orientada a la } \\
\text { búsqueda de } \\
\text { ideas } \\
\text { relacionadas } \\
\text { con el modelo, } \\
\text { pero sin filtros } \\
\text { (lo que } \\
\text { encuentran no } \\
\text { siempre es } \\
\text { apropiado en } \\
\text { función de la } \\
\text { edad) }\end{array}$ & $\begin{array}{l}\text { Orientada a la } \\
\text { búsqueda de ideas } \\
\text { relacionadas con el } \\
\text { modelo que sean útiles } \\
\text { para validar las } \\
\text { pruebas obtenidas y, } \\
\text { por tanto, adecuadas a } \\
\text { lo que los alumnos } \\
\text { pueden comprender. }\end{array}$ \\
\hline $\begin{array}{l}\text { Comprobación y } \\
\text { comunicación } \\
\text { argumentada de } \\
\text { las } \\
\text { interpretaciones y } \\
\text { conclusiones }\end{array}$ & $\begin{array}{l}\text { Afirmaciones } \\
\text { sin } \\
\text { argumentación. }\end{array}$ & $\begin{array}{l}\text { No se conecta } \\
\text { con las } \\
\text { predicciones } \\
\text { o hipótesis } \\
\text { planteadas y } \\
\text { tampoco se } \\
\text { tienen en } \\
\text { cuenta las } \\
\text { pruebas ni las } \\
\text { ideas teóricas } \\
\text { de referencia. }\end{array}$ & $\begin{array}{l}\text { Se parte de las } \\
\text { predicciones o } \\
\text { hipótesis, pero } \\
\text { las } \\
\text { interpretaciones } \\
\text { y conclusiones } \\
\text { no se basan en } \\
\text { el modelo } \\
\text { teórico de } \\
\text { referencia. }\end{array}$ & $\begin{array}{l}\text { Se parte de hablar de } \\
\text { la comprobación de las } \\
\text { predicciones o } \\
\text { hipótesis y se incide en } \\
\text { las pruebas recogidas y } \\
\text { se concluye en base al } \\
\text { modelo. }\end{array}$ \\
\hline $\begin{array}{l}\text { Motivación e } \\
\text { implicación del } \\
\text { alumnado }\end{array}$ & $\begin{array}{l}\text { Alumnos } \\
\text { motivados por } \\
\text { la diferencia } \\
\text { entre este tipo } \\
\text { de actividad y } \\
\text { las anteriores. }\end{array}$ & $\begin{array}{l}\text { Los alumnos } \\
\text { se muestran } \\
\text { participativos } \\
\text { e intervienen } \\
\text { aportando } \\
\text { ideas. }\end{array}$ & $\begin{array}{l}\text { Surgen retos } \\
\text { individuales y } \\
\text { hay discusión en } \\
\text { el marco de } \\
\text { pequeños } \\
\text { grupos y del } \\
\text { gran grupo. }\end{array}$ & $\begin{array}{l}\text { Hay trabajo autónomo } \\
\text { combinado con el del } \\
\text { grupo. Manifiestan } \\
\text { muchas iniciativas. }\end{array}$ \\
\hline $\begin{array}{l}\text { Autoevaluación y } \\
\text { metacognición }\end{array}$ & $\begin{array}{l}\text { Esperan que el } \\
\text { docente les } \\
\text { indique si lo } \\
\text { están haciendo } \\
\text { bien. }\end{array}$ & $\begin{array}{l}\text { Aunque se } \\
\text { propone } \\
\text { alguna } \\
\text { reflexión, es } \\
\text { superficial y } \\
\text { mecanicista. }\end{array}$ & $\begin{array}{l}\text { Se utilizan } \\
\text { estrategias } \\
\text { cooperativas } \\
\text { para promover } \\
\text { la } \\
\text { autoevaluación } \\
\text { y reflexión en } \\
\text { relación con } \\
\text { algunas } \\
\text { estrategias } \\
\text { básicas } \\
\text { asociadas a la } \\
\text { indagación } \\
\text { científica. }\end{array}$ & $\begin{array}{l}\text { Aplicación de } \\
\text { estrategias diversas } \\
\text { orientadas a pensar y } \\
\text { regular la planificación } \\
\text { de la acción y los } \\
\text { criterios de evaluación, } \\
\text { de forma que } \\
\text { promuevan la } \\
\text { autorregulación de la } \\
\text { práctica indagatoria. }\end{array}$ \\
\hline
\end{tabular}

Analizando el progreso en relación con cada indicador se observan los siguientes cambios y dificultades: 
- En todo el proceso se evidencia que inicialmente los docentes no le dan demasiada importancia al contexto escogido y se centran en los conceptos que quieren enseñar. Acaban seleccionando alguno, pero inicialmente lo perciben como pérdida de tiempo que se resta al aprendizaje de conocimientos y dicen: “¿Con diez minutos es suficiente, ¿no?”. Su visión es la de "motivar", y continuar explicando de forma tradicional. En un paso siguiente, lo utilizan para que los alumnos se planteen preguntas y surjan ideas previas, pero luego se olvida, y no se promueve que las nuevas ideas los alumnos las construyan y relacionen con datos provenientes del análisis de situaciones cotidianas y reales. Por ejemplo, las preguntas sobre las plantas surgieron en un recorrido por el jardín de la escuela, pero no se volvió a visitarlo para buscar pruebas de las ideas y predicciones expresadas, a partir de observar, identificar y cuantificar. Es cierto que trabajando en contexto nos es fácil transferir lo aprendido a otras situaciones (Gilbert, 2006; King, 2012), pero la génesis de las ideas se asocia a un ejemplo real (Brown, Collins y Duguid, 1989) y así lo manifiestan los mismos maestros que, a la pregunta por algún contenido bien consolidado, siempre responden pensando en su relación con un contexto concreto. Esta metarreflexión les ayuda a revisar su concepción sobre la importancia del contexto.

- En referencia a las preguntas, inicialmente los maestros explican que habitualmente las plantean ellos y que se fijan en si la respuesta dada por los alumnos es cierta o no. Después se dan cuenta de lo enriquecedor que es que respondan primero a nivel individual, activando sus conocimientos previos, y luego discutan y completen sus respuestas en equipo. Un dato que confirma el cambio es que han asumido como práctica habitual de aula "contar hasta diez" antes de aceptar respuestas de nadie. También ha mejorado el tipo de preguntas planteadas, y son más acordes al modelo que quieren ayudar a construir. Por ejemplo, pasan de preguntar "¿Cómo se reproducen las plantas? a ¿Qué pasaría si no existieran las abejas?" O de "¿Cuáles son los estados de la materia? a ¿Cómo podemos (en qué forma) encontrar agua en la Naturaleza?”. Además, todos promovieron que los alumnos reflexionaran sobre qué pensaban y hacían al experimentar, aplicando la "base de orientación" que se había consensuado en las sesiones de formación. El paso a discutir con ellos sus preguntas y regular si son "buenas", se evidenció que era mucho más complejo (Gómez et al., 2004).

- Las primeras actividades experimentales que nos explican los maestros, las proponen sin dejar que los alumnos participen en su diseño. Más adelante, dejan en manos de los estudiantes la planificación de algunas experiencias y éstos, en muchas ocasiones, buscan prácticas muy espectaculares que los docentes creen motivadoras, pero de las que son incapaces de explicar las observaciones a través de su modelo. Por otro lado, no se piensa en diferentes variables, ni en cómo controlarlas. Están lejos de asumir que la investigación ha de posibilitar recoger pruebas de las ideas previas expresadas, o de las predicciones o hipótesis planteadas.

- Los datos que se promueve que los alumnos recojan son, hasta ahora, fundamentalmente de tipo cualitativo. Al inicio son de observaciones poco sistemáticas, y en el estadio siguiente lo son algo más, de manera que posibilitan compararlos. A medida que se ha ido ganando confianza en conocimientos de ciencias, los maestros empiezan a promover la recogida de datos cuantitativos, y en su organización a través de gráficos. Por ejemplo, inicialmente sólo observaban que el agua se evaporaba al calentarla, mientras que, en una etapa posterior, a partir de datos recogidos mediante un termómetro y un cronómetro, construyeron la gráfica $\mathrm{T} / \mathrm{t}$ y estimaron la temperatura de ebullición.

- La visión de modelo teórico y de modelización no son conceptos y prácticas que formen parte del perfil inicial de los maestros al enseñar ciencias. A partir de sus percepciones iniciales del sentido del término "modelo", se discutió su significado y su importancia en la ciencia. Reflexionamos sobre las características de una práctica científica escolar que podríamos considerar auténtica, y se habló de la interrelación entre la indagación, la modelización y la 
argumentación en la escuela y en el quehacer científico real (Couso, 2014). Son ideas realmente complejas y en un estadio inicial, apenas se representan que las ideas alternativas que expresan los alumnos responden a modelos que habrán de evolucionar. En algunos casos se proponen tenerlas en cuenta. Por ejemplo, al indagar sobre los diferentes volúmenes ocupados por líquidos y sólidos un grupo de alumnos argumenta: "Después de jugar con las fichas de dómino (actividad para ayudar a la modelización en la que construian estructuras con las fichas) hemos visto que teniendo el mismo número de fichas las estructuras eran diferentes. Eso nos ha hecho ver porque el nivel del aceite sube cuando se descongela y baja el del agua. Porqué las estructuras con las partículas que los forman son diferentes", pero no es el caso más general, ya que los maestros tienden a aplicar su rutina de "explicar las ideas verdaderas" de la ciencia para que los aprendices las repitan.

- En relación al uso de bibliografía, al inicio se animaba a los niños y niñas a buscar informaciones en libros o en Internet sin ninguna orientación específica. Los maestros decían que los niños "investigaban para encontrar las respuestas" y se daba por buena cualquier referencia al tema objeto de estudio, aunque no fuera ni significativa ni fácilmente interpretable. Un ejemplo de frase consultada fue: "por encima de la temperatura crítica, si comprimimos un gas obtendremos un fluido supercrítico y no un líquido". Posteriormente fueron más orientadas o se hablaba de si se entendían, pero no se discutía su posible relevancia para validar ideas previas o para interpretar las observaciones realizadas.

- Al escribir las conclusiones, en la mayoría de los casos, inicialmente los alumnos se limitan a afirmaciones sin ningún tipo de argumentación (descriptivas). En un nuevo estadio, los maestros los animan a generar argumentos, pero sin ayudarles a que los relacionen con sus predicciones y los fundamenten en los datos recogidos. En el estadio dos, estimulan establecer estas conexiones, pero aún no promueven que los interpreten en base a un posible modelo teórico de referencia. Se ha constatado que algunos maestros empiezan a promover que las explicaciones estén basadas explícitamente en el modelo o ideas teóricas construidas y algunos de sus alumnos pasan (al hablar de cambios en los estados de la materia) de escribir "cuando se enfría un gas se vuelve líquido" a "el vapor de agua se condensa en la tapa de la olla porque las particulas pierden velocidad al chocar con ella". - Respecto a la motivación interna, que conlleva poner en juego los propios sentimientos, emociones y valores y, también, las capacidades que posibilitan tanto el trabajo autónomo como con los demás, los maestros centran su valoración en que los alumnos muestran interés y participan. Estas percepciones son especialmente relevantes en las primeras actividades de indagación propuestas, pero poco a poco van valorando otras variables, como el hecho de que observan que sus alumnos van adquiriendo confianza en las propias capacidades de pensamiento, de acción y de comunicación.

- Finalmente, los cambios en relación a la evaluación de tipo metacognitivo, representa para los maestros un giro muy amplio respecto a su práctica habitual. Inicialmente devuelven los trabajos con breves comentarios, y es lo que esperan los alumnos. En un estadio posterior, las reflexiones son más específicas ("las predicciones iniciales que habéis planteado ban sido interesantes"), pero las genera el docente. Algunos de ellos, realizan un nuevo paso y empiezan a promover que los alumnos sean los que generen estos comentarios, en actividades de coevaluación en el marco de los equipos de trabajo a través de sencillas rúbricas que se han consensuado previamente. Aún así, faltaría estimular que los fundamenten y, muy especialmente, que sugieran posibles propuestas razonadas para avanzar, ya que diversos trabajos (Hinojosa y Sanmartí, 2015; Rosa y Alves Filho, 2014; Schraw et al., 2006) muestran la importancia de trabajar los procesos metacognitivos con los alumnos, ayudándolos a tomar consciencia de su conocimiento y de sus estructuras conceptuales.

No todos los docentes progresaron al mismo ritmo, a pesar de que la reflexión sobre las ideas y sobre las prácticas fue compartida. Dos de las maestras se mostraron capaces de ayudar a los 
compañeros a pensar en algunas estrategias didácticas: "siempre hay que exigirles -a los alumnos- que den las explicaciones utilizando el modelo". Los indicadores en los que más cuesta progresar son el diseño de la investigación y la explicación-argumentación en base al modelo, que son las cuestiones sobre las que los maestros plantean mas reparos y dudas.

Destacaríamos que la visión del conocimiento científico como conjunto de modelos teóricos que interrelacionan ideas y experiencias, y que a partir de ellos se pueden predecir y explicar muchos fenómenos, está muy lejos de las concepciones de muchos docentes y ello explica en buena parte las dificultades del cambio. Por el contrario, está muy interiorizada la creencia de que la ciencia es un conjunto de afirmaciones atomizadas (definiciones), y que se demuestra que se aprende si se sabe repetir cada una de ellas de forma aislada. Y tampoco se concibe que su aprendizaje tenga relación con recoger pruebas y argumentar a partir de aplicar procesos de indagación para validar ideas previas, predicciones o hipótesis, ya que el experimento se percibe como una actividad que sirve para visualizar una afirmación y no para reconstruirla.

Cambiar estas concepciones es muy complejo, ya que en todo caso se cree que los alumnos aprenderán las ideas a partir de observar, sin más. Pero concebir el aprendizaje de las ciencias como un proceso de modelización basado en la indagación (Windschitl et al., 2008), condiciona que se perciba el contexto como algo necesario para posibilitar la expresión de las ideas que se habrán de poner a prueba (y no sólo como motivación), que se valore que no todas las preguntas son igualmente válidas para promover un proceso de construcción del modelo desde su complejidad y un razonamiento significativo, que la función que se dé a la observación y experimentación se relacione con la evolución de las ideas iniciales y la obtención de pruebas a utilizar en las explicaciones y su argumentación, y que la evaluación se aplique para promover la autorregulación metacognitiva de este proceso (Romero-Ariza, 2017).

\section{Reflexiones finales}

En toda propuesta de formación permanente es importante que los profesores asuman e interioricen qué se entiende por práctica científica y, por tanto, apliquen procesos de cambio que se van discutiendo y consensuando a partir de compartir ideas, prácticas y reflexiones. Se requiere un cierto grado de compromiso hacia el cambio, pero éste sólo es sostenible si se da en el marco de un equipo de trabajo. Partir del análisis de la propia práctica comporta que poco a poco los docentes sean más creativos y busquen romper con su rutina, de manera que se van autoajustando a una visión más indagatoria de la práctica científica escolar. Ellos mismos advierten su progreso y explicitan: "No trabajábamos así, pero va de fábula" (cuaderno de campo). Y si el proceso es colectivo, con compañeros de profesión, posibilita que en momentos de desánimo (que los hay) alguien promueva no abandonar -siempre que el clima de la formación posibilite que se expresen las dificultades sin percibirlas como algo negativo-.

Como señala Imbernón (2007) la formación debe tener en cuenta que, más que "actualizar" a un docente y enseñarle, se deben crear las condiciones para que innove y reflexione sobre qué sucede al aplicar la innovación, por qué sucede y qué nuevas mejoras se podrían introducir. Y ello requiere generar ambientes para que todo el mundo se pueda expresar, preservando su autoestima, y gestionar las posibles emociones negativas que sin duda van surgiendo. Y si, como en nuestro caso, esas condiciones se dan en equipos de trabajo naturales, posiblemente la probabilidad de que sus consecuencias sean más relevantes y se mantengan en el tiempo es mucho más alta, ya que es el grupo el que avanza, aunque no necesariamente todos los miembros al mismo ritmo.

De acuerdo con otras investigaciones (Jiménez, Aragón y Oliva, 2017; Solís et al., 2013), el proceso de cambio profesional es lento y exige pasar por etapas "intermedias", en las que el 
profesorado va apropiándose del significado de "indagar" y de su relación con el proceso de modelización. La evolución es el resultado de discutir desde las percepciones y prácticas de cada docente y, al mismo tiempo, de la aportación de nuevos referentes teóricos desde la didáctica de las ciencias que ayuden a ponerlas en duda.

Así pues, y al mismo tiempo, se está construyendo en los equipos de trabajo de profesores y en el centro una nueva cultura profesional. Estamos avanzando en el desarrollo individual y del grupo a través de la experimentación y reflexión, en y sobre la práctica, delimitando los problemas y estableciendo el asesoramiento y apoyo necesario desde la didáctica de la ciencia. Este planteamiento de la formación como modelización de la práctica profesional, es de hecho coherente con el proceso de modelización del conocimiento científico que se discute en su seno. Y como es sabido y apuntan los mismos maestros "aprendemos más meditando sobre cómo hacemos las cosas que atendiendo cómo nos explican cuáles son las buenas prácticas”.

\section{Agradecimientos}

Investigación realizada en el marco del grupo LiEC financiada por el Ministerio de Economía y Competitividad (referencia PGC2018-096581-B-C21). El Grup LiEC forma parte del grupo LiCEC (referencia 2014SGR1492). A la Escola Pia Sarrià-Calassanç.

\section{Referencias}

Brown, J.S., Collins, A., \& Duguid, P. (1989). Situated Cognition and the Culture of Learning. Educational Researcher, 18(1), 32-42.

Capps, D., Crawford, B. \& Constas, M. (2012). A Review of Empirical Literature on Inquiry Professional Development: Alignment with Best Practices and a Critique of the Findings. Journal of Science Teacher Education, 23(3), 291-318. https://doi.org/10.1007/s10972-012-92752

Chinn, A. C. \& Malhotra, A. B. (2002). Epistemologically authentic inquiry in schools. A theoretical framework for evaluating Inquiry task. Science Education, 86(2), 175-218.

Coenders, F. y Terlouw, C. (2015). A Model for In-service Teacher Learning in the Context of an Innovation. Journal of Science Teacher Education, 26(5), 451-470.

Coll, C. (1994). Psicologia y Curriculum. Una aproximación psicopedagógica a la elaboración del currículum escolar. Barcelona: Paidós.

Copello, M.I. y Sanmartí, N. (2001). Fundamentos de un modelo de formación permanente del profesorado de ciencias centrado en la reflexión dialógica sobre las concepciones y las prácticas. Enseñanza de las Ciencias, 19(2), 269-283.

Couso, D. (2014). De la moda de "aprender indagando" a la indagación para modelizar: una reflexión crítica. XXVI Encuentro de Didáctica de las Ciencias Experimentales (Huelva).

Esteve, O. (2013). Entre la praáctica y la teoriía. Comprender para actuar. Ikastaria, 19, 13-36.

Garrido, A. y Couso, D. (2017). La modelización en la formación inicial de maestros. ¿Qué mecanismos o estrategias la promuevem? Enseñanza de las Ciencias, Núm. Extra, 137-143.

Gómez, A., Márquez, C., Pujol, R.M. y Sardá, A. (2004). La construcción de modelos explicativos complejos mediante preguntas mediadoras. Investigación en la Escuela, 53, 71-82.

Hargreaves, A. (1999). Hacia una geografía social de la profesión docente. En A. Pérez Gómez, J. Barquín y J. F. Angulo (Eds.), Desarrollo profesional del docente: Política, investigación y práctica (119145). Madrid: Akal. 
Hinojosa, J. y Sanmartí, N. (2015). La autorregulación metacognitiva como medio para facilitar la transferencia en mecánica. Revista Eureka sobre Enseñanza y Divulgación de las Ciencias, 12(2), 249263.

Hinojosa, J. y Sanmartí, N. (2016). Indagando en el aula de ciencias: primeros pasos. En J. Sánchez Martín y F. Cañada, Cañana (Eds.), Ciencias para comprender el mundo: Investigación e innovación en didáctica de las ciencias experimentales (pp. 119-128). Madrid: Entimema.

Imbernón, F. (2007). La formación permanente del profesorado. Nuevas ideas para formar en la innovación y el cambio. 10 ideas clave. Barcelona: Graó.

Jiménez, N., Aragón, L. y Oliva, J. M. (2017). Progresión en las visiones epistemológicas de estudiantes de especialidades de ciencias del máster en profesorado de secundaria. Enseñanza de las Ciencias, Núm. Extra, 2307-2312.

Jimenez-Aleixandre, M.P. (2000). Modelos didácticos. En: J. Perales y P. Cañal. (Eds.). Didáctica de las Ciencias: Teoría y Práctica de la Enseñanza de las Ciencias. Alcoy: Marfil.

King, D. (2012). New perspectives on context-based chemistry education: using a dialectical sociocultural approach to view teaching and learning, Studies in Science Education, 48(1), 51-87.

Mellado, V., Ruiz, C., Bermejo, M. L. \& Jiménez-Pérez, R. (2006). Contributions from the philosophy of science to the education of science teachers. Science \& Education, 15(5), 419-445.

Osborne, J. (2014). Teaching scientific Practices: Meeting the Challenge of Change. Journal of Science Teacher Education, 25(2), 177-196.

Porlán, R., Martín del Pozo, R., Rivero, A., Harres, J., Azcárate, P. y Pizzato, M. (2010). El cambio del profesorado de ciencias I: marco teórico y formativo. Enseñanza de las Ciencias, 28(1), 31-46.

Porlán, R., Rivero, A. y Solís, E. (2011). Un modelo de formación para el cambio del profesorado de ciencias. Actas Primeras Jornadas de Innovación Docente de la Facultad de Ciencias de la Educación de la Universidad de Sevilla, 1-9.

Porlán, R. (2016). Un programa para la formación docente del profesorado universitario en activo basado en los "ciclos de mejora". Mesa redonda en XXVII Encuentro de Didáctica de las Ciencias Experimentales, Badajoz.

Romero-Ariza M. (2017). El aprendizaje por indagación, ¿existen suficientes evidencias sobre sus beneficios en la enseñanza de las ciencias? Revista Eureka sobre Enseñanza y Divulgación de las Ciencias 14(2), 286-299.

Rosa, C. W. y Alves Filho, J. P. (2014). Estudo da viabilidade de uma proposta didática metacognitiva para as atividades experimentais em física. Ciência y Educação (Bauru), 20(1), 61-81.

Simarro, C., Couso, D. y Pintó, R. (2013). Indagació basada en la modelització: un marc per al treball pràctic. Ciències, 25, 35-43.

Sanmartí, N. (2002). Didáctica de las ciencias en la educación secundaria obligatoria. Madrid: Síntesis Educación.

Schraw, G., Crippen, K.J. \& Hartley, K. (2006). Promoting Self-Regulation in Science Education: Metacognition as Part of a Broader Perspective on Learning. Research in Science Education, 36, 111-139.

Schwarz, C., Reiser, B, Davis, B., Kenyon, L, Acher, A., Fortus, D., Hug, B. \& Krajcik, J. (2009). Designing a learning progression of scientific modeling: Making scientific modeling accessible and meaningful for learners. Journal of Research in Science Teaching, 46(6), 632-654.

Solís, E., Martín del Pozo, R., Rivero, A. y Porlán, R. (2013). Expectativas y concepciones de los estudiantes del MAES en la especialidad de Ciencias. Revista Eureka sobre Enseñanza y Divulgación de las Ciencias, 10 (num. extraordinario), 496-513. 
Valcárcel, M.V. y Sánchez, G. (2000). La formación del profesorado en ejercicio. En F. J. Perales y P. Cañal. (Eds.), Didáctica de las Ciencias: Teoría y Práctica de la Enseñanza de las Ciencias. Alcoy: Marfil.

Vázquez-Bernal, B., Jiménez-Pérez, R. y Mellado, V. (2007). El desarrollo profesional del profesorado de ciencias como integración de la reflexión y la práctica. La hipótesis de la complejidad. Revista Eureka sobre Enseñanza y Divulgación de las Ciencias, 4(3), 372-393.

Vázquez-Bernal, B., Mellado, V., Jiménez-Pérez, R. y Martos, M. (2013). La evaluación como objeto de investigación. El caso de una profesora de química de enseñanza secundaria. Educación Quimica, 24(3), 335-342.

Windschitl, M., Thompson, J. \& Braaten, M. (2008). Beyond the scientific method: model-based inquiry as a new paradigm of preference for school science investigations. Science Education, 92(5), 941-967.

\section{Información sobre los autores}

Autor: Julià Hinojosa Lobato

Institución: Departamento de Didáctica de las Matemáticas y las Ciencias Experimentales. Universidad Autónoma de Barcelona

Grupo de investigación LiEC

Email: julia.hinojosa@escolapia.cat

ORCID: 0000-0001-6596-1564

Autora: Neus Sanmartí i Puig

Institución: Departamento de Didáctica de las Matemáticas y las Ciencias Experimentales. Universidad Autónoma de Barcelona

Grupo de investigación LiEC

Email: neussanmarti@gmail.com

ORCID: 0000-0002-9229-563X 


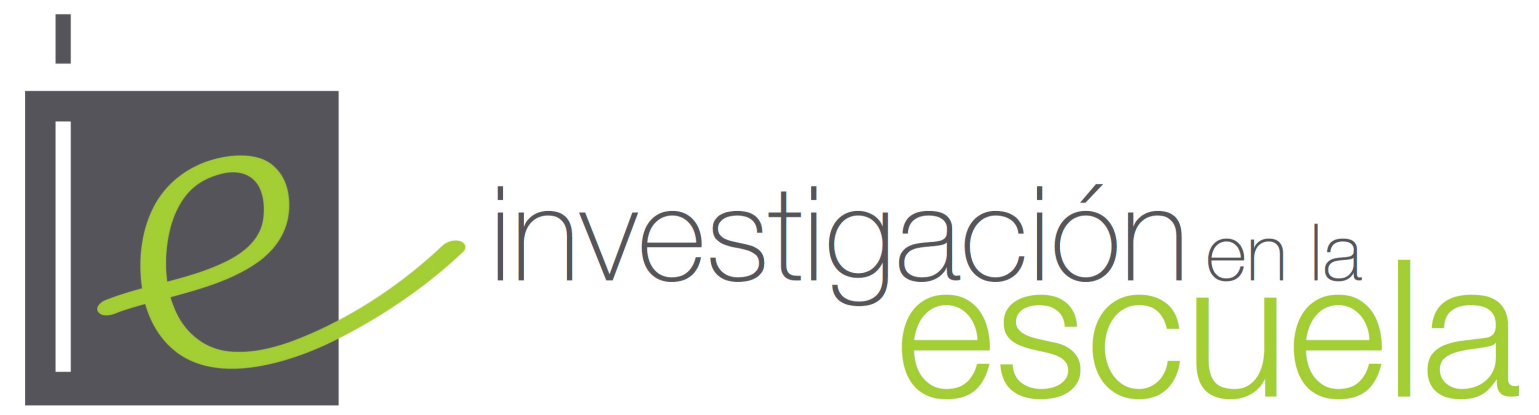

Revista académica evaluada por pares y de acceso abierto

Número 99

30 de diciembre de 2019

ISSN 2443-9991

\section{(c) (i) (2)}

EY Es Esta obra está bajo una licencia Creative Commons. Los/as lectores/as pueden compartir, copiar y redistribuir el material en cualquier medio o formato, así como adaptar, remezclar, transformar y construir a partir del material para cualquier propósito, incluso comercialmente. Para ello, deben de hacerlo bajo los siguientes términos: dando crédito de forma adecuada, brindando un enlace a la licencia e indicando si se han realizado cambios. Si se remezcla, transforma o crea a partir del material, debe distribuir su contribución bajo la misma licencia del original.

Más detalles de la licencia de CreativeCommons se encuentran en https://creativecommons.org/licenses/by-sa/4.0/deed.es

Cualquier otro uso debe ser aprobado en conjunto por el autor/es, o Investigación en la Escuela.

ư

Revista Editada por la Universidad de Sevilla. https://editorial.us.es/es/revistainvestigacion-en-la-escuela

Por errores y sugerencias contacte a secretaria@investigacionenlaescuela.es

La revista Investigación en la Escuela desde su origen en 1987 hasta su no 87 (2015) fue editada por Díada Editora. 
